\title{
Distribution of the total angular momentum in relativistic configurations
}

\author{
Michel Poirien * \\ CEA - Paris-Saclay University, Laboratory "Interactions, Dynamics, \\ and Lasers", CE Saclay, F-91191 Gif-sur-Yvette, France \\ Jean-Christophe Pain \\ CEA, DAM, DIF, F-91297 Arpajon, France and \\ Université Paris-Saclay, CEA, Laboratoire Matière en Conditions Extrêmes, F-91680 Bruyères-le-Châtel, France
}

(Dated: Tuesday $6^{\text {th }}$ July, 2021)

\begin{abstract}
This paper is devoted to the analysis of the distribution of the total angular momentum in a relativistic configuration. Using cumulants and generating function formalism this analysis can be reduced to the study of individual subshells with $N$ equivalent electrons of momentum $j$. An expression as a nth-derivative is provided for the generating function of the $J$ distribution and efficient recurrence relations are established. It is shown that this distribution may be represented by a Gram-Charlier-like series which is derived from the corresponding series for the magnetic quantum number distribution. The numerical efficiency of this expansion is fair when the configuration consists of several subshells, while the accuracy is less good when only one subshell is involved. An analytical expression is given for the odd-order momenta while the even-order ones are expressed as a series which provides an acceptable accuracy though being not convergent. Such expressions may be used to obtain approximate values for the number of transitions in a spin-orbit split array: it is shown that the approximation is often efficient when few terms are kept, while some complex cases require to include a large number of terms.
\end{abstract}

\section{INTRODUCTION}

In order to model the emission and absorption spectral properties of hot plasmas, in the context of stellar physics or in laser-plasma experiments, for inertial-confinement-fusion studies for instance, one has to properly describe multielectron configurations with several open subshells. In particular, knowing a priori the number of lines between two configurations is of great interest. The statistics of electric-dipole (E1) lines was investigated by Moszkowski [1], Bauche and Bauche-Arnoult [2], and more recently by Gilleron and Pain [3]. The number of lines is a cornerstone of opacity codes, in order to decide whether a transition array can be modeled statistically, using the above-mentioned methods, or needs a detailed-line accounting calculation, involving the diagonalization of the Hamiltonian [4, 5]. Alternative methods such as the partially-resolved-transition-array approach [6 -8], and its extension to the superconfiguration formalism [9, 10], can be applied when the number of lines of a transition array exceeds a particular value. The statistics of electric quadrupole (E2) lines was also studied [11].

In counting problems, the generating-function technique is a powerful tool, whether to obtain analytical expressions, to derive recursion relations, or to find approximate formulas. The generating function leads also to the determination of cumulants, which are an important ingredient of statistical modeling and from which the moments may be obtained. In this framework, we recently published analytical formulas and recurrence relations for the number of electronic configurations in a superconfiguration [12], together with a statistical analysis based on the calculation of cumulants.

The determination of the total angular momentum multiplicities was first investigated by nuclear physicists [13] in the framework of the shell model [14, 15] and later by atomic physicists for electronic configurations. Considering a system of $N$ identical fermions, the problem boils down to deducing the allowed total angular momenta $J$ to which they may couple. Some values of $J$ are forbidden by anti-symmetrization due to the Pauli exclusion principle, some others occur more than once. As pointed out by Condon and Shortley, the number $Q(J)$ of levels with angular momentum $J$ is equal to the number of states with projection $M=J$ minus the number of states with $M=J+1$. In his 1936 paper [13], Bethe modeled the distribution of angular momentum by the Wigner-type distribution which can be deduced from a Gaussian form of the distribution $P(M)$. Years later, Bauche and Bauche-Arnoult derived analytical formulas for $Q(J)$ and for the number of lines between two electronic configurations, assuming a fourthorder Gram-Charlier expansion series of $P(M)$ [2]. At the same period, Hirst and Wybourne used statistical group theory to determine the parameters of the Wigner-type approximate formula and pointed out a connection with the theory of partitions of integers [16].

\footnotetext{
* michel.poirier@cea.fr

$\dagger$ jean-christophe.pain@cea.fr
} 
In a previous work [17], cited here as paper I, we proposed a statistical analysis of the distribution $P(M)$ in the case of a relativistic configuration (i.e., made of subshells $j^{N}$ ). Using the generating-function formalism and the properties of Gaussian binomial coefficients, we derived recursion relations for $P(M)$ as well as analytical expressions for the cumulants. In the same work, we carried out an analysis of the distribution $P(M)$ using Gram-Charlier and Edgeworth expansion series at any order.

However, although $Q(J)$ can be deduced from $P(M)$, only very few properties of $Q(J)$ are explicitly known. The aim of the present paper is multiple. First, following our analysis of the magnetic quantum number distribution, we propose here an expression for the generating function of the distribution $Q(J)$, from which we derive efficient recurrence relations. Second, using paper I analysis, we generalize the above-mentioned Bethe formula in the form of a Gram-Charlier-like series the convergence of which is analyzed. Third, we propose expressions for the moments $\sum_{J}(2 J+1)^{n} Q(J)$, in a closed analytical form for odd $n$, and as a cumulant-based expansion for even $n$, with a study of its convergence. This sheds some light on the delicate question about the number of levels $\sum_{J} Q(J)$ inside a given relativistic configuration. Another useful application of this formalism is that it allows one to derive the number of lines inside a transition array, which is a significant information when analyzing the complex spectra such as those obtained in high-temperature plasmas.

\section{GENERATING FUNCTION AND RECURRENCES}

\section{A. Population distribution of the total angular momentum}

In the most general relativistic configuration, the determination of the distribution of the total angular momentum $Q(J)$ is connected to the distribution of the magnetic quantum number $P(M)$ through the relation [2]

$$
\begin{gathered}
Q\left(J_{\max }\right)=P\left(J_{\max }\right) \\
Q(J)=P(M=J)-P(M=J+1)
\end{gathered}
$$

where in the latter equation one has $0 \leq J<J_{\max }$. However for the sake of analyticity, it is very important to allow for any value of $J$ between $-J_{\max }$ and $J_{\max }-1$ in Eq.(II.1b), keeping in mind that the "unphysical" $Q(J)$ are convenient mathematical tools. Using the symmetry property $P(-M)=P(M)$ one easily verifies from the property (II.1b) imposed on any $J$ that

$$
Q(-J-1)=P(-J-1)-P(-J)=-(P(J)-P(J+1))=-Q(J)
$$

for any $J \leq J_{\max }$. This means that $Q(J)$ is an odd function versus the origin $J_{0}=-1 / 2$. In other words if $N$ is odd $Q(-1 / 2)=0, Q(-3 / 2)=-Q(1 / 2) \ldots$, if $N$ is even $Q(-1)=-Q(0), Q(-2)=-Q(1) \ldots$ Finally for $J<-J_{\max }-1$ or $J>J_{\max }, Q(J)$ vanishes. One must notice that from such a definition, one has

$$
\sum_{J=-J_{\max }-1}^{J_{\max }} Q(J)=0
$$

which prevents us from normalizing the $Q(J)$ distribution.

\section{B. Analytical expression of the generating function and recurrences}

In this subsection we consider relativistic configurations consisting of a single subshell with $N$ equivalent electrons $j^{N}$. In paper I, we have shown that the distribution $P(M)$ can be obtained from the generating function $-z$ being the arbitrary complex variable of this function -

$$
\sum_{M} P(M) z^{M}=z^{-J_{\max }} \prod_{p=1}^{N} \frac{z^{2 j+2-p}-1}{z^{p}-1}=z^{-J_{\max } \mathscr{F}(j, N ; z)}
$$

where $\mathscr{F}(j, N ; z)$ is the product $\prod_{p=1}^{N}\left(z^{2 j+2-p}-1\right) /\left(z^{p}-1\right)$ also known as the Gaussian binomial coefficient. From the general property (II.1b) of the distribution $Q$ and from the above relation (II.4) one gets at once

$$
z^{J_{\max }+1} \sum_{J} Q(J) z^{J}=z^{J_{\max }+1} \sum_{J}(P(J)-P(J+1)) z^{J}=(z-1) z^{J_{\max }} \sum_{M} P(M) z^{M}
$$


which proves that the generating function for $Q(J)$ is simply equal to the generating function for $P(M)$ multiplied by $(z-1)$. One will note that this property holds for any relativistic configuration, containing one or several subshells.

This important results bears a series of consequences. The first one is that the numbers $Q(J)$ may be obtained as a simple nth-derivative when a single subshell $j^{N}$ is involved. Similarly to Eq. (II.4) one has

$$
\mathscr{G}(j, N ; z)=\sum_{n=0}^{2 J_{\max }+1} Q\left(n-J_{\max }-1\right) z^{n}=(z-1) \prod_{p=1}^{N} \frac{z^{2 j+2-p}-1}{z^{p}-1} .
$$

This allows one to express the population $Q(J)$ as a multiple derivative

$$
Q\left(n-J_{\max }-1\right)=\left.\frac{1}{n !} \frac{d^{n}}{d z^{n}}\left[(z-1) \prod_{p=1}^{N} \frac{z^{2 j+2-p}-1}{z^{p}-1}\right]\right|_{z=0}
$$

where $n$ varies from 0 to $2 J_{\max }+1$. From the symmetry property (II.2) one has also

$$
Q\left(n-J_{\max }-1\right)=-Q\left(J_{\max }-n\right)
$$

which allows one to obtain $Q(J)$ for positive $J$ with less derivative operations.

Recurrence relations can be derived in a similar manner as in paper I. One may use the identity straightforwardly derived from the generating function (II.6)

$$
\mathscr{G}(j, N ; z)\left(z^{N}-1\right)=\mathscr{G}(j, N-1 ; z)\left(z^{2 j+2-N}-1\right)
$$

and derive $n$ times using the Leibniz rule. One may also use the relation on the $M$ distribution obtained in paper I (with some rewriting)

$$
P(M-N ; j, N)-P(M ; j, N)=P(M-j-1 ; j, N-1)-P(M+j+1-N ; j, N-1)
$$

— the indices $j, N$ are added when necessary for a correct understanding — and the fundamental relation (II.1b), which gives the recurrence property

$$
Q(J-N ; j, N)-Q(J ; j, N)=Q(J-j-1 ; j, N-1)-Q(J+j+1-N ; j, N-1) .
$$

With the definition

$$
\mathscr{Q}_{j, N}(n)=Q(n-N(2 j+1-N) / 2-1 ; j, N)
$$

one writes the somewhat simpler formula

$$
\mathscr{Q}_{j, N}(n-N)-\mathscr{Q}_{j, N}(n)=\mathscr{Q}_{j, N-1}(n-2 j-2+N)-\mathscr{Q}_{j, N-1}(n) .
$$

The recurrence is initialized by the $N=1$ value

$$
Q(J ; j, N=1)=\delta_{J, j}-\delta_{J,-j-1} .
$$

In order to avoid the consideration of the full range of $n$ values one may use the symmetry property (II.2).

Accordingly, a recurrence relation on $j$ is obtained by changing $j$ by $1 / 2$ while the number of electrons $N$ is kept constant. From

$$
\mathscr{G}(j+1 / 2, N ; z)\left(z^{2 j+2-N}-1\right)=\mathscr{G}(j, N ; z)\left(z^{2 j+2}-1\right)
$$

and multiple derivation using Leibniz rule, or from the paper I relation

$$
P(M-2 j-1+N ; j, N)-P(M ; j, N)=P\left(M-2 j-1+\frac{N}{2} ; j-\frac{1}{2}, N\right)-P\left(M+\frac{N}{2} ; j-\frac{1}{2}, N\right),
$$

one gets the second recurrence relation

$$
Q(J-2 j-1+N ; j, N)-Q(J ; j, N)=Q\left(J-2 j-1+\frac{N}{2} ; j-\frac{1}{2}, N\right)-Q\left(J+\frac{N}{2} ; j-\frac{1}{2}, N\right) .
$$


With the $\mathscr{Q}_{j, N}$ quantities one has

$$
\mathscr{Q}_{j, N}(n-2 j-1+N)-\mathscr{Q}_{j, N}(n)=\mathscr{Q}_{j-1 / 2, N}(n-2 j-1)-\mathscr{Q}_{j-1 / 2, N}(n) .
$$

The recurrence is initialized using

$$
Q(J ; j=0, N)=\delta_{J, 0}-\delta_{J,-1} .
$$

One notes that the same recurrence relations formally hold for $P(M)$ and $Q(J)$. Of course the solutions for $P(M)$ and $Q(J)$ differ because the initial values $(N=1$ or $j=0)$ are different for $P$ and $Q$. It has been shown in paper I that such relations allow one to get the full set of $P(M)$ with a minimum of computations. Of course the same statement holds for the derivation of the $Q(J)$ distribution.

\section{Application to the analysis of even-odd staggering in angular-momentum distribution}

It has been shown by Bauche and Cossé [18] and Pain [19] that when the number of electrons $N$ in a subshell is even, the levels with even angular momentum $\sum_{J \text { even }} Q(J)$ outnumber the levels with odd angular momentum $\sum_{J \text { odd }} Q(J)$. This effect was proven to exist for non-relativistic as well as relativistic configurations. Expressions were derived in Ref. [18] for the excess $\sum_{J \text { even }} Q(J)-\sum_{J \text { odd }} Q(J)$ by an explicit counting of the levels. As shown in Ref. 19] and in Appendix @ the present generating-function formalism allows for a simpler derivation of the excess evaluation by computing $\mathscr{G}(j ; N ;-1)$. Moreover, other sum rules may also be obtained by computing this generating function at other points on the trigonometric circle.

\section{APPROXIMATION BY GRAM-CHARLIER-LIKE SERIES}

\section{A. Analytical formulation}

The generating function for the $J$-momentum distribution (II.6) being straightforwardly derived from the generating function (II.4) for the magnetic quantum number, one might expect a similar property to hold when transposing the cumulant generating function to the $Q(J)$ distribution. However one must note that the standard definition for this generating function $K(t)$

$$
\exp (K(t))=\langle\exp (t M)\rangle=\sum_{M} P(M) e^{t M} / \sum_{M} P(M)
$$

expresses it as the logarithm of a sum, and that this sum is normalized by $\sum_{M} P(M)$. As mentioned above (II.3) the sum of the $Q(J)$ vanishes. Therefore a standard Gram-Charlier analysis is precluded here. A workaround consists in using the Gram-Charlier series for the $P(M)$ distribution and evaluating the difference $P(J)-P(J+1)$. In paper I we obtained the expansion

$$
P_{\mathrm{GC}}(M)=\frac{G}{\left(2 \pi \sigma^{2}\right)^{1 / 2}} \exp \left(-\frac{M^{2}}{2 \sigma^{2}}\right) \sum_{n} c_{2 n} H e_{2 n}\left(\frac{M}{\sigma}\right)
$$

where $G$ is the degeneracy factor, which is, for the most general relativistic configuration $j_{1}^{N_{1}} \cdots j_{w}^{N_{w}}$

$$
G=\prod_{s=1}^{w}\left(\begin{array}{c}
2 j_{s}+1 \\
N_{s}
\end{array}\right)
$$

and $\sigma$ is the standard deviation for the $M$-distribution

$$
\sigma^{2}=\sum_{s=1}^{w} \frac{j_{s}+1}{6} N_{s}\left(2 j_{s}+1-N_{s}\right)
$$

In Eq. (III.2a) $H e_{2 n}(X)$ is the Chebyshev-Hermite polynomial [20]

$$
H e_{N}(X)=(-1)^{N} e^{X^{2} / 2} \frac{d^{N}}{d X^{N}}\left(e^{-X^{2} / 2}\right)=N ! \sum_{m} \frac{(-1)^{m} X^{N-2 m}}{2^{m} m !(N-2 m) !} .
$$


The expression for the coefficients $c_{2 n}$ is detailed below. The distribution for $J$ is therefore given by the finite difference formula, assuming the series is convergent

$$
Q_{\mathrm{GC}}(J)=P_{\mathrm{GC}}(J)-P_{\mathrm{GC}}(J+1)=-\sum_{p=0}^{\infty} \frac{2^{-2 p}}{(2 p+1) !} \frac{\partial^{2 p+1}}{\partial J^{2 p+1}} P_{\mathrm{GC}}(J+1 / 2) .
$$

The multiple derivative in the above formula is obtained from the explicit expression (III.2a) and from the property of Hermite polynomials, easily obtained from the above relation (III.3),

$$
\frac{d^{p}}{d X^{p}}\left(e^{-X^{2} / 2} H e_{n}(X)\right)=(-1)^{p} e^{-X^{2} / 2} H e_{n+p}(X)
$$

Putting such expression in the expansion (III.2a) we get

$$
\begin{aligned}
Q_{\mathrm{GC}}(J) & =P_{\mathrm{GC}}(J)-P_{\mathrm{GC}}(J+1) \\
& =\frac{G}{(2 \pi)^{1 / 2} \sigma^{2}} \exp \left(-\frac{(J+1 / 2)^{2}}{2 \sigma^{2}}\right) \sum_{p=0}^{\infty} \frac{(2 \sigma)^{-2 p}}{(2 p+1) !} \sum_{n=0}^{\infty} c_{2 n} H e_{2 n+2 p+1}((J+1 / 2) / \sigma) .
\end{aligned}
$$

This can be recast as a Gram-Charlier-like formula

$$
Q_{\mathrm{GC}}(J)=\frac{G}{(2 \pi)^{1 / 2} \sigma^{2}} \exp \left(-\frac{(J+1 / 2)^{2}}{2 \sigma^{2}}\right) \sum_{m=0}^{\infty} d_{2 m+1} H e_{2 m+1}((J+1 / 2) / \sigma)
$$

with

$$
d_{2 m+1}=\sum_{\substack{n, p \\ n+p=m}} \frac{(2 \sigma)^{-2 p}}{(2 p+1) !} c_{2 n}
$$

The coefficients $d_{2 m+1}$ may be obtained by two methods. As a general property of the Gram-Charlier expansion (see, e.g., Ref.[12]), the even-order coefficients $c_{2 n}$ can be expressed versus the cumulants of the $M$-distribution

$$
c_{2 n}=\sum_{\substack{a_{4}, a_{6} \cdots a_{2 n} \\ 2 a_{4}+3 a_{6}+\cdots+n a_{2 n}=n}} \frac{1}{a_{4} !}\left(\frac{\kappa_{4}}{4 ! \sigma^{4}}\right)^{a_{4}} \frac{1}{a_{6} !}\left(\frac{\kappa_{6}}{6 ! \sigma^{6}}\right)^{a_{6}} \cdots \frac{1}{a_{2 n} !}\left(\frac{\kappa_{2 n}}{(2 n) ! \sigma^{2 n}}\right)^{a_{2 n}} .
$$

The interest of this formula is that the cumulants $\kappa_{2 n}$ have a fairly simple expression as shown in paper I

$$
\kappa_{2 k}=\frac{B_{2 k}}{2 k}\left[\sum_{p=1}^{N}(2 j+2-p)^{2 k}-\sum_{p=1}^{N} p^{2 k}\right]
$$

where $B_{2 k}$ are the even-order Bernoulli numbers. In addition, when several subshells are involved, their cumulants are additive. The second method using an alternative expression for coefficients $c_{2 n}$ is studied in Appendix B.

Using the relation (III.9), one checks that the $d_{2 m+1}$ coefficient is written as a sum over the indices $\left(p, a_{4}, \ldots a_{2 n}\right)$ with the constraints $p+n=m, 2 a_{4}+3 a_{6}+\ldots n a_{2 n}=n$. A thorough analysis of this set of indices shows that, after a convenient regrouping of terms, the sum is indeed equal to

$$
\begin{aligned}
d_{2 m+1} & =\sum_{\substack{p, a_{4} \cdots a_{2 m} \\
p+2 a_{4}+\cdots m a_{2 m}=m}} \frac{(2 \sigma)^{-2 p}}{(2 p+1) !} \frac{1}{a_{4} !}\left(\frac{\kappa_{4}}{4 ! \sigma^{4}}\right)^{a_{4}} \cdots \frac{1}{a_{2 m} !}\left(\frac{\kappa_{2 m}}{(2 m) ! \sigma^{2 m}}\right)^{a_{2 m}} \\
& =\frac{1}{\sigma^{2 m}} \sum_{\substack{p, a_{4} \cdots a_{2 m} \\
p+2 a_{4}+\cdots m a_{2 m}=m}} \frac{2^{-2 p}}{(2 p+1) !} \frac{1}{a_{4} !}\left(\frac{\kappa_{4}}{4 !}\right)^{a_{4}} \cdots \frac{1}{a_{2 m} !}\left(\frac{\kappa_{2 m}}{(2 m) !}\right)^{a_{2 m}} .
\end{aligned}
$$

In the above sum, the set $\left(p, a_{4}, \cdots a_{2 m}\right)$ generates all the partitions of the number $m$, as expressed by the condition $p+2 a_{4}+\cdots+m a_{2 m}=m$. 
The first coefficients $d_{2 m+1}$ are

$$
\begin{aligned}
d_{1} & =1 \\
d_{3} & =\frac{1}{24 \sigma^{2}} \\
d_{5} & =\frac{1}{1920 \sigma^{4}}+\frac{\kappa_{4}}{24 \sigma^{4}} \\
d_{7} & =\frac{1}{322560 \sigma^{6}}+\frac{\kappa_{4}}{576 \sigma^{6}}+\frac{\kappa_{6}}{720 \sigma^{6}}
\end{aligned}
$$

which can be easily evaluated using the expression of the cumulants for the distribution $P(M)$ given by Eq. (III.10). One easily checks that accounting only for the first term $d_{1}$ in the series provides the above-mentioned Bethe approximation [13] for the $J$ distribution

$$
Q_{\text {Bethe }}(J)=\frac{G}{\sigma^{3} \sqrt{2 \pi}}(J+1 / 2) e^{-\frac{(J+1 / 2)^{2}}{2 \sigma^{2}}} .
$$

The expression (III.11a for the $d_{2 m+1}$ coefficients as a function of cumulants is formally simple, however it is also useful to get an alternative form as a function of the moments $\mu_{k}=\sum_{M} M^{k} P(M) / \sum_{M} P(M)$. The relevant expression is derived in the Appendix B.

\section{B. Accuracy criterion}

As in paper I, one may define various convergence criteria for the obtained Gram-Charlier-like formula. Writing $Q_{\mathrm{GC}}(J ; k)$ for the series (III.7) truncated at index $k$ (with $k$ odd), the global absolute error is

$$
\Delta_{\mathrm{abs}}(k)=\left[\sum_{J=J_{\min }}^{J_{\max }}\left(Q_{\mathrm{GC}}(J ; k)-Q(J)\right)^{2} /\left(J_{\max }-J_{\min }+1\right)\right]^{1 / 2}
$$

where $J_{\min }$ is 0 or $1 / 2$, and the global relative error

$$
\Delta_{\mathrm{rel}}(k)=\left[\sum_{J=J_{\min }}^{J_{\max }}\left(Q_{\mathrm{GC}}(J ; k) / Q(J)-1\right)^{2} /\left(J_{\max }-J_{\min }+1\right)\right]^{1 / 2} .
$$

However, while in paper I the $P(M)$ distribution was always positive on its definition range, the number $Q(J)$ may vanish for a series of $J$ values. In the relative error computation (III.15), these values will not be included. Hence the absolute value (III.14) is here a better accuracy criterion.

\section{VARIOUS TESTS OF THE GRAM-CHARLIER-LIKE DISTRIBUTION}

\section{A. Single-subshell configuration}

Let us first consider a configuration with a small angular momentum and number of electrons. On Fig. 1(a) we have plotted the exact and Gram-Charlier-like approximation for the $J$ distribution for two $j=5 / 2$ electrons. One has then $Q(J)=1$ for $J=0,2,4$ and $Q(J)=0$ for $J=1,3$. It is not expected that a Gram-Charlier-like expansion provides a reasonable approximation of such a distribution. Indeed, we state that a series with few terms provides a very poor approximation of $Q(J)$. Quite unexpectedly, we note that including about 24 terms in the expansion, the Gram-Charlier-like formula gives a reasonable approximation of $Q(J)$. Adding more terms, one notes that the quality of the agreement deteriorates gradually. In order to analyze the series behavior, we have plotted on Fig. 1(b) the average absolute error (III.14) as a function of the number of terms kept in series (III.7), e.g., 3 terms corresponds to contributions up to $d_{5}$, and $m$ terms to contributions up to $d_{2 m-1}$. The average relative error is not plotted here since two $Q(J)$ vanish. The absolute error is also shown in the $J=0$ and $J=4$ cases. We note that the average absolute error is usually about 0.6. This quite large value is not surprising since for configurations with few electrons the present statistical treatment is not expected to be efficient. A more unexpected result is that the error for both $J=0$ and $J=4$ cases is small when 23 terms are accounted for. Some more information is brought by the analysis of 


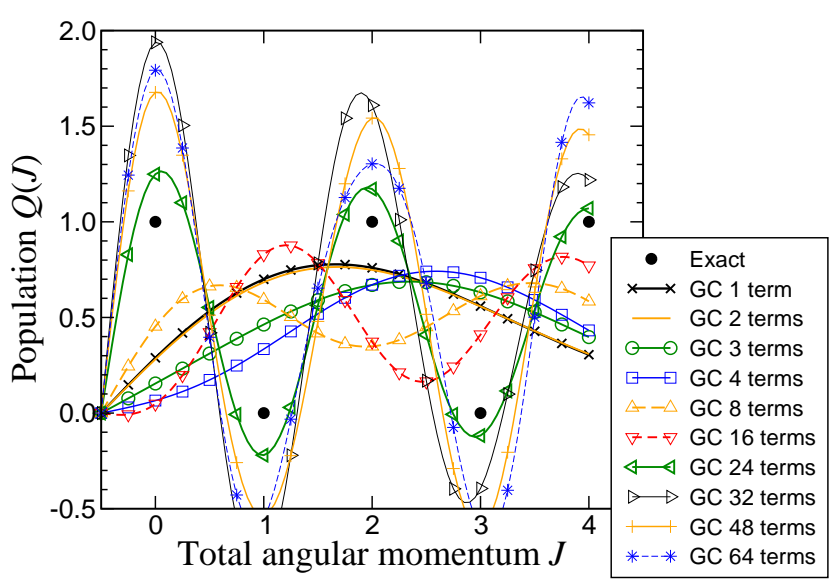

(a) Exact and Gram-Charlier-like approximation for the total angular momentum distribution $Q(J)$.

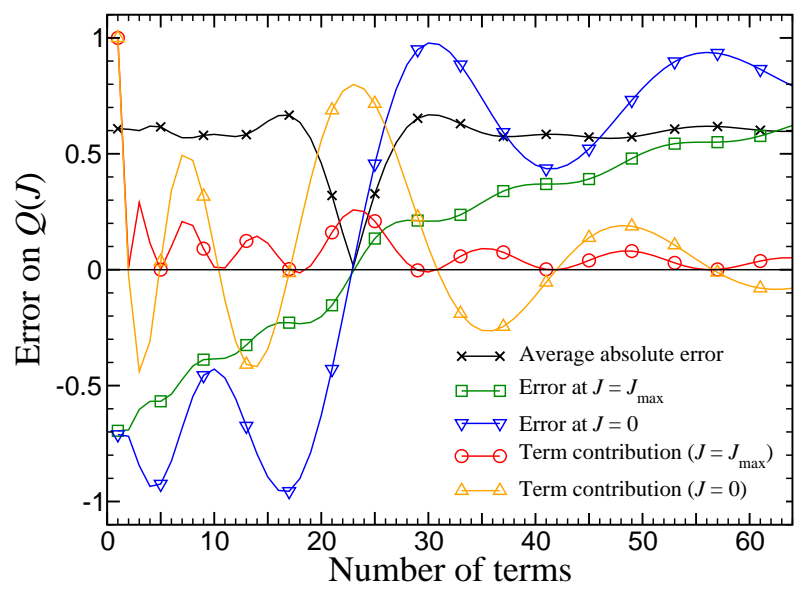

(b) Error done using the Gram-Charlier-like approximation for the total angular momentum distribution $Q(J)$. The abscissa is the number of terms kept in the expansion (III.2a). The contribution of each term to the sum, normalized to the first term, is also shown.

FIG. 1. Analysis of the total angular momentum distribution $Q(J)$ in the relativistic configuration with one subshell $j_{1}=$ $5 / 2, N_{1}=2$.

the relative contribution of each term, i.e. the ratio $\rho(m)=d_{2 m-1} H e_{2 m-1}((J+1 / 2) / \sigma) / d_{1} H e_{1}((J+1 / 2) / \sigma)$ which is also plotted on Fig. 1(b) for $J=0$ and $J=4$. It appears that this ratio is large for 23 terms $(2 m+1=45)$, which corresponds to the optimum index in the sum. Above this local maximum the term contribution decreases but slowly, as a numerical analysis shows that the series is not convergent.

The case of a configuration with one subshell and more electrons is illustrated by two examples in Appendix C It is shown that an acceptable representation of the distribution $Q(J)$ is obtained by a Gram-Charlier-like expansion with few terms.

\section{B. Configuration with a small and a large angular momentum}

As a second example, let us consider the $J$ distribution in the configuration $j_{1}=5 / 2, N_{1}=2, j_{2}=37 / 2, N_{2}=1$ which is analogous to the case considered in Ref. [3]. It has been shown in paper I that the $M$ distribution exhibits a wide plateau for $-29 / 2 \leq M \leq 29 / 2$, which can be approximated by a Gram-Charlier expansion provided a large number of terms is kept. Accordingly the $J$ distribution is characterized by a rather sharp peak close to $J=37 / 2$ while all other $Q(J)$ values outside this region cancel. Such a stepwise function is certainly very difficult to reproduce with a Gram-Charlier-like expansion. Indeed this can be observed on Fig. 2(a) where we have plotted various GramCharlier-like approximations versus the exact $Q(J)$ values. It appears that the Gram-Charlier-like expansions with one and two terms are indistinguishable at the drawing accuracy. Looking at the definitions (III.12), one may estimate the first-two-terms ratio

$$
\rho(2)=d_{3} H e_{3}((J+1 / 2) / \sigma) / d_{1} H e_{1}((J+1 / 2) / \sigma) \simeq(J+1 / 2)^{2} /\left(24 \sigma^{4}\right),
$$

and for instance at the peak $J=37 / 2$, using $\sigma^{2}=1499 / 12$ obtained from Eq. (III.2C) this ratio is $\rho \simeq 9.6 \times 10^{-4}$. This property holds indeed whatever the configuration analyzed. Looking at the various truncated expansions on Fig. 2(a) it appears that the three-term expansion including the excess kurtosis $\kappa_{4}$ is here a poor approximation of the $Q(J)$ distribution. Including several tens of terms in the expansion, it turns out that the series (III.7) "converges" toward the correct value. However, this appears to be a "best approximation" rather than a convergence, since adding several hundreds of terms in the series we noted that the absolute error levels off at $\simeq 0.2$ and does not tend to 0 .

The absolute error as defined by (III.14) is plotted in Fig. 2(b) as well as the error done on $J_{\min }=1 / 2$ and $J_{\max }=45 / 2$. As mentioned above, the approximations with 1 or 2 terms are almost identical. One notes that including high-order terms improves the validity of the Gram-Charlier-like expansion. However, the convergence is slow, with a rather limited accuracy obtained even when 30 terms are accounted for. This statement is in agreement with what was mentioned in paper I for the $P(M)$ distribution in the same configuration. 


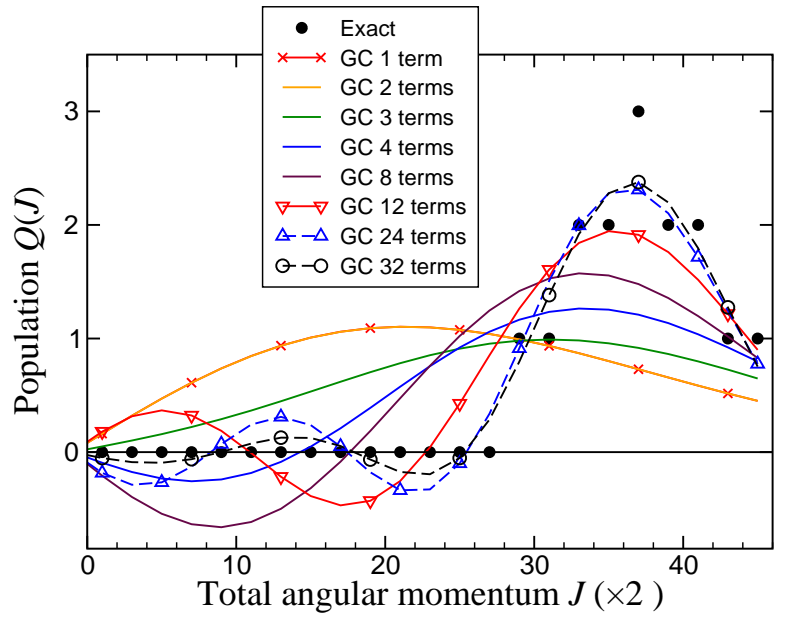

(a) Exact and Gram-Charlier-like approximation for the total angular momentum distribution $Q(J)$.

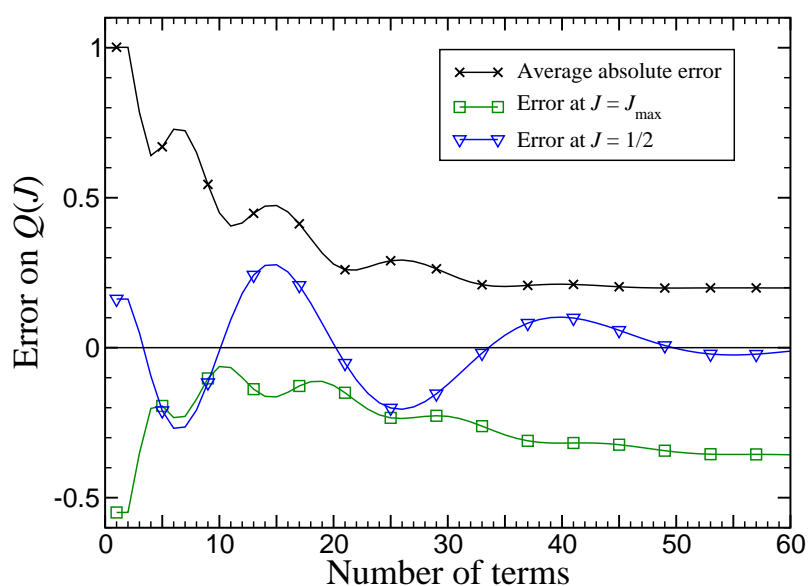

(b) Error done using the Gram-Charlier-like approximation for the total angular momentum distribution $Q(J)$. The abscissa is the number of terms kept in the expansion (III.2a).

FIG. 2. Analysis of the total angular momentum distribution $Q(J)$ in the relativistic configuration with 2 subshells $j_{1}=$ $5 / 2, N_{1}=2, j_{2}=37 / 2, N_{2}=1$.

\section{Several half-filled subshells}

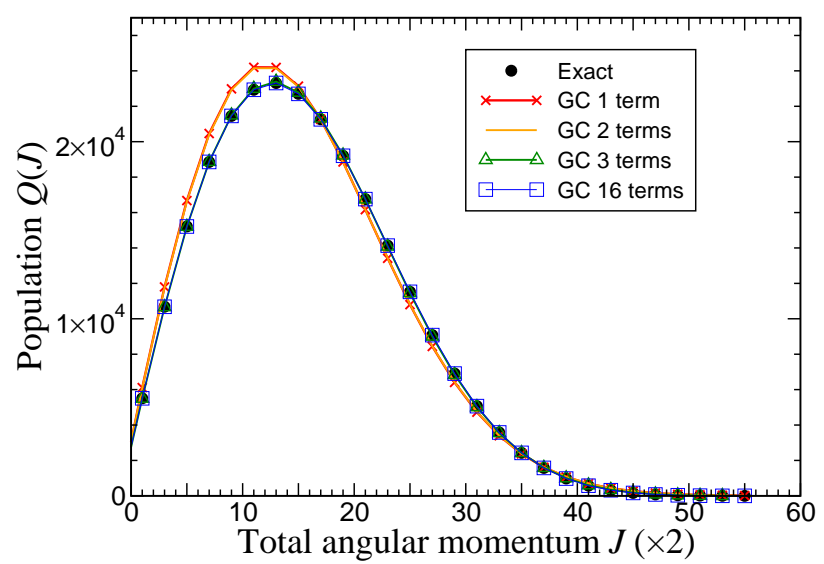

(a) $J$ distribution: exact and Gram-Charlier-like approximation. The Gram-Charlier-like expansion includes various terms in the sum III.2a. The 1 -term and 2 -term approximations are indistinguishable at the drawing accuracy, as well as the various approximations with more than 2 terms.

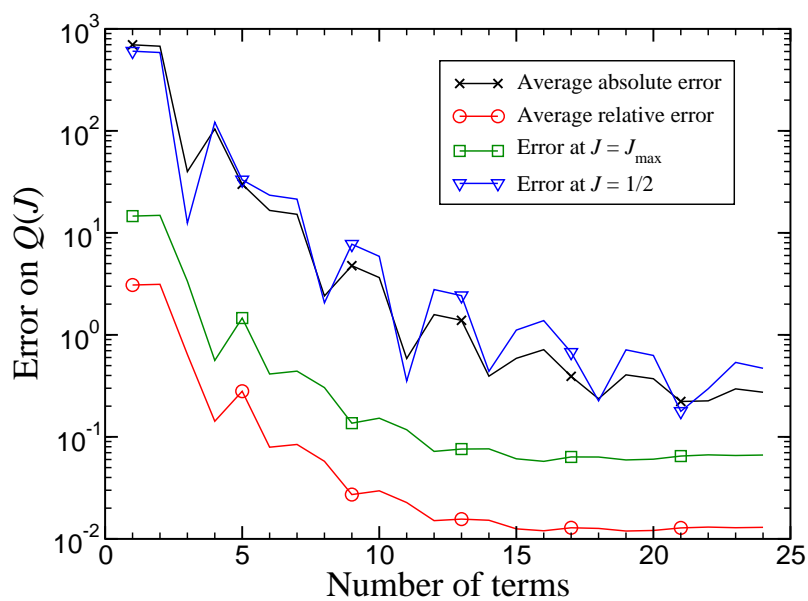

(b) Error done using the Gram-Charlier-like approximation. The average absolute and relative errors are defined in main text. The error at $J_{0}=J_{\max }$ or $J_{0}=1 / 2$ is the value of the unsigned difference $\left|Q_{\mathrm{GC}}\left(J_{0} ; k_{\max }\right)-Q\left(J_{0}\right)\right|$ plotted as a function of the number of terms $\left(k_{\max }+1\right) / 2$, where $k_{\max }$ is the truncation index in sum (III.2a).

FIG. 3. Exact and Gram-Charlier-like approximation for the total angular momentum $Q(J)$ in the relativistic configuration with 5 half-filled subshells $j=i-1 / 2, N_{i}=j_{i}+1 / 2, i=1-5$.

From the central-limit theorem and from paper I analysis we expect that the present Gram-Charlier-like approximation will perform better when subshells contain a larger number of electrons. To this respect, we have plotted in Fig. 3(a) the exact values of the populations $Q(J)$ and the approximations obtained with expansion (III.2a) with various numbers of terms. Only the positive part $J>0$ is represented, but of course exact and approximate distribution laws verify the rule $Q(-J-1)=-Q(J)$. One observes here that the Gram-Charlier-like approximation with 3 terms, including the excess kurtosis as shown by Eq. (III.12C), is indeed a quite good approximation of the distribution 
$Q(J)$. As mentioned above the one- and two-term forms of the expansion III.2a are almost equal since the ratio (IV.1) is again small: $1 / 24 \sigma^{2}=1 / 1010$. Fig. 3(b) is a plot of various expressions of the error as a function of the number of terms retained in the expansion (III.2a). This second part of Fig. 3 shows that, while including 3 terms in the expansion is an acceptable approximation, much better results may be obtained with more terms, the optimum being reached with about 12 terms. Though a mathematical analysis of the convergence of the series (III.2a) would be outside the scope of this work, we may conclude that such expansion is of asymptotic nature.

\section{Configuration with a large number of subshells}

As in paper I, we consider here the distribution $Q(J)$ in the case of a configuration with 10 subshells $j=1 / 2-$ $19 / 2$, all containing a single electron. For this 10 -electron configuration one has $J_{\max }=50$, the degeneracy is $2^{10} .10 !=3.7158912 \times 10^{9}$, and the population $P(M)$ varies on 8 orders of magnitude $-Q(J)$ varying on 7 orders of magnitude. We have plotted in Fig. 4 the $Q(J)$ distribution computed exactly and the Gram-Charlier-like expansions truncated at various orders. One observes once again that the approximations including one or two terms are very similar, and that in this case both differ hardly from the exact value. Conversely, as was observed for the $M$ distribution [17], the expansions including at least 3 terms, i.e., the kurtosis contribution or term $d_{5}$ in Eq.(III.11a), provide a fair representation of the angular momentum distribution on the whole range. As seen on Fig. 4(c), the 3-term approximation brings a significant improvement with respect to the Bethe formula (1-term approximation), while at least 6 terms are necessary to get a better accuracy. The average error versus the number of terms is plotted on Fig. 4(c) which shows the accuracy slowly improves with the number of terms.

In order to confirm the non-convergent character of the Gram-Charlier expansion, we performed several GramCharlier-expansion studies on configurations with $N$ singly-populated subshells $j_{i}=i-1 / 2, i$ from 1 to $N, N_{i}=1$ for each subshell. For $N=5$, we observed that the smallest relative error III.15 was reached for 92 terms in the series, with an error $\Delta_{\text {rel }}=3 \times 10^{-5}$. For $N=6$ (resp. 8), the minimum occurs for 148 (resp. 334) terms and reaches $\Delta_{\text {rel }}=10^{-5}$ (resp. $\left.3 \times 10^{-7}\right)$. Beyond these optimal values, the error tends to increase. We therefore note that the larger the number of electrons, the later the onset of the divergence, and the better the quality of the Gram-Charlier expansion. A similar behavior occurs for the Stirling expansion for the factorial. Such series are called asymptotic and are studied in various treatises [21].

\section{APPLICATION TO THE COMPUTATION OF MOMENTS AT ANY ORDER}

In this section, we consider the case of a single-subshell configuration $j^{N}$. The present conclusions may be generalized to the multiple-subshell case by taking benefit of the additivity of cumulants - the value $\kappa_{2 k}$ for a single subshell is to be replaced by the sum of the contributions of individual subshells. The formula (III.7) is useful to get the odd-order moments, i.e, the average of $(2 J+1)^{2 n+1}$ on the distribution $Q(J)$. The first odd-order moment, that will be used as the normalization factor, is simply equal to the total degeneracy of the configuration

$$
\sum_{J=J_{\min }}^{J_{\max }}(2 J+1) Q(J)=\left(\begin{array}{c}
2 j+1 \\
N
\end{array}\right)=G
$$

In order to evaluate the higher odd-order moments, one uses the classical inversion property of the Hermite polynomials - easily derived using the Rodrigues formula (III.3) and repeated integration by parts -

$$
X^{k}=k ! \sum_{m=0}^{\lfloor k / 2\rfloor} \frac{H e_{k-2 m}(X)}{2^{m} m !(k-2 m) !}
$$

where $\lfloor x\rfloor$ is the integer part of $x$, and the orthogonality property $[20]$

$$
\int_{-\infty}^{+\infty} d X \exp \left(-X^{2} / 2\right) H e_{m}(X) H e_{n}(X)=\delta_{n m} \sqrt{2 \pi} n !
$$

which allows us to get directly the average values $\left\langle H e_{2 n+1}(Q+1 / 2)\right\rangle$ on the Gram-Charlier-like distribution (III.7). Replacing the discrete sum over $J$ by an integral $\int_{-1 / 2}^{\infty}$ where the symmetry property $Q_{\mathrm{GC}}(-J-1)=-Q_{\mathrm{GC}}(J)$ 


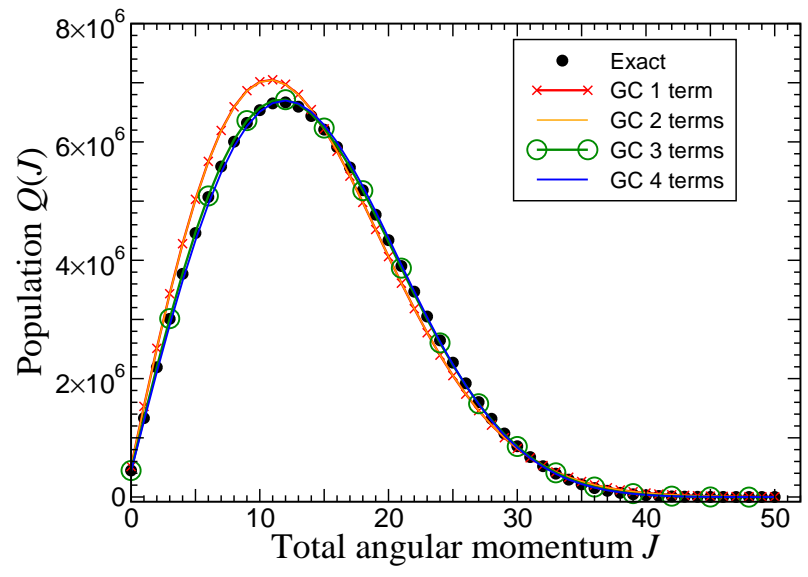

(a) $J$ distribution: exact and Gram-Charlier-like approximation

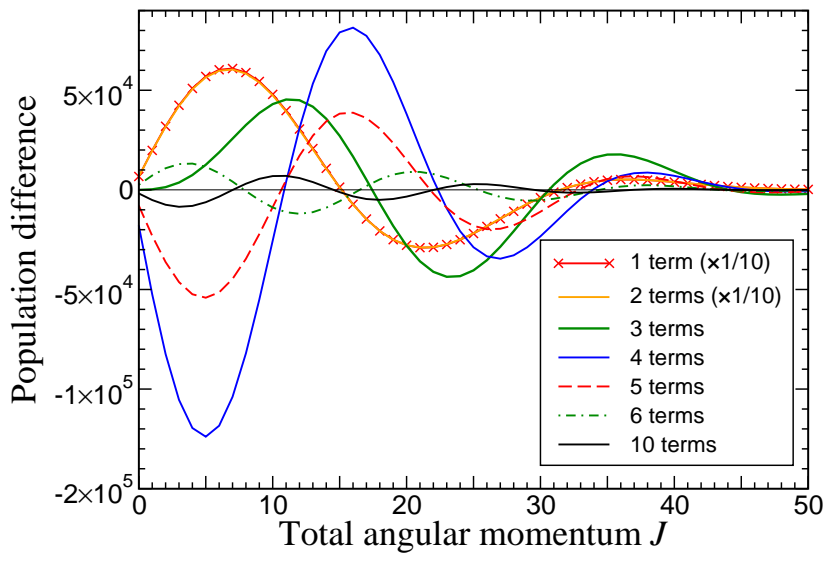

(b) Difference between the populations at various orders and exact value. For the first two cases, the difference is divided by 10 .

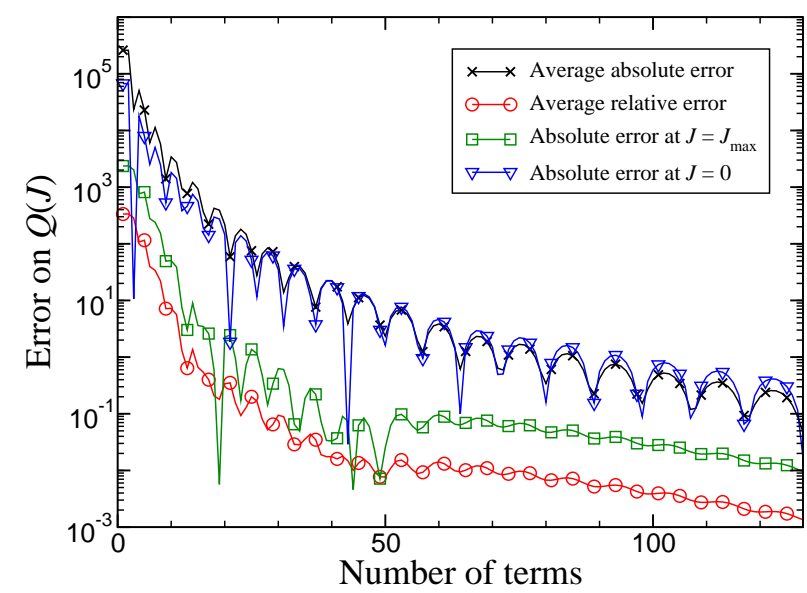

(c) Error done on $Q(J)$ as a function of the number of terms kept in the expansion [III.7). See Fig. 3(b) caption for details.

FIG. 4. Exact and Gram-Charlier-like approximation for the total angular momentum distribution $Q(J)$ in the relativistic configuration with 10 subshells $j_{i}=i-1 / 2, i=1-10$ all singly populated.

(meaning that the function $Q_{\mathrm{GC}}(J)$ is odd versus the origin $J=-1 / 2$ ) is used, one gets at once

$$
\begin{aligned}
\sum_{J} H e_{2 n+1}((J+1 / 2) / \sigma) Q(J) & \simeq \int_{-1 / 2}^{+\infty} d J Q_{\mathrm{GC}}(J) H e_{2 n+1}((J+1 / 2) / \sigma) \\
& =\frac{1}{2} \int_{-\infty}^{+\infty} d J Q_{\mathrm{GC}}(J) H e_{2 n+1}((J+1 / 2) / \sigma) \\
& =\frac{1}{2} \frac{G}{\sigma}(2 n+1) ! d_{2 n+1}
\end{aligned}
$$


where the $d_{2 n+1}$ are known as a function of the cumulants using Eq. (III.11a). In some cases one can even show that the equality (V.4a) is indeed not approximate but exact. Using the inversion formula (V.2), one has also

$$
\begin{aligned}
\left.\sum_{J}((J+1 / 2) / \sigma)\right)^{2 n+1} Q(J) & \simeq \int_{-1 / 2}^{+\infty} d J Q_{\mathrm{GC}}(J)((J+1 / 2) / \sigma)^{2 n+1} \\
& =\frac{1}{2} \frac{G}{\sigma}(2 n+1) ! \sum_{m=0}^{n} \frac{d_{2 m+1}}{2^{m} m !}
\end{aligned}
$$

More generally, using the formula

$$
\int_{0}^{\infty} y^{p} e^{-y^{2} / 2} H e_{n}(y) d y=\frac{\Gamma(p+1) 2^{(n-p-1) / 2} \sqrt{\pi}}{\Gamma(1+(p-n) / 2)}
$$

which can be derived using the formula (III.3) and repeated integration by parts, one may easily obtain an approximation for the sum $\left.\sum_{J \geq 0}((2 J+1) / \sigma)\right)^{n} Q(J)$ including for even $n$. Explicitly, one gets

$$
M_{n}=\sum_{J \geq 0}(2 J+1)^{n} Q(J) \simeq \int_{-1 / 2}^{\infty}(2 J+1)^{n} Q_{\mathrm{GC}}(J) d J=2 G(2 \sigma)^{n-1} n ! \sum_{m \geq 0} \frac{2^{m-(n+1) / 2}}{\Gamma\left(\frac{n+1}{2}-m\right)} d_{2 m+1} .
$$

If $n$ is odd, the above sum terminates at $m=(n-1) / 2$. Moreover, one can prove that the relation (V.7) is not approximate but exact at least concerning odd orders for which convergence is not an issue. The first odd-order moments are

$$
\begin{aligned}
& M_{1}=G \\
& M_{3}=G\left(1+12 \sigma^{2}\right) \\
& M_{5}=G\left(1+40 \sigma^{2}+240 \sigma^{4}+80 \kappa_{4}\right) \\
& M_{7}=G\left(1+84 \sigma^{2}+1680 \sigma^{4}+6720 \sigma^{6}+560\left(1+12 \sigma^{2}\right) \kappa_{4}+448 \kappa_{6}\right) .
\end{aligned}
$$

Various examples of $M_{n}$ values at even and odd order $n$ are presented in Table \

\begin{tabular}{cccccccc}
\hline \hline$n$ & Exact & 1 term & 2 terms & 3 terms & 4 terms & 8 terms & 16 terms \\
\hline 0 & 468 & 483.094 & 482.869 & 468.728 & 466.171 & 468.715 & 468.513 \\
1 & 11440 & 11440 & 11440 & 11440 & 11440 & 11440 & 11440 \\
2 & 348584 & 344929 & 345090 & 348456 & 348821 & 348575 & 348585 \\
3 & $1.226368 \times 10^{7}$ & $1.225224 \times 10^{7}$ & $1.226368 \times 10^{7}$ & $1.226368 \times 10^{7}$ & $1.226368 \times 10^{7}$ & $1.226368 \times 10^{7}$ & $1.226368 \times 10^{7}$ \\
4 & $4.785009 \times 10^{8}$ & $4.925592 \times 10^{8}$ & $4.932490 \times 10^{8}$ & $4.788311 \times 10^{8}$ & $4.783097 \times 10^{8}$ & $4.785042 \times 10^{8}$ & $4.785008 \times 10^{8}$ \\
5 & $2.020396 \times 10^{10}$ & $2.187025 \times 10^{10}$ & $2.191109 \times 10^{10}$ & $2.020396 \times 10^{10}$ & $2.020396 \times 10^{10}$ & $2.020396 \times 10^{10}$ & $2.020396 \times 10^{10}$ \\
6 & $9.080727 \times 10^{11}$ & $1.055062 \times 10^{12}$ & $1.057525 \times 10^{12}$ & $9.031082 \times 10^{11}$ & $9.086918 \times 10^{11}$ & $9.080691 \times 10^{11}$ & $9.080727 \times 10^{11}$ \\
7 & $4.293402 \times 10^{13}$ & $5.465375 \times 10^{13}$ & $5.480684 \times 10^{13}$ & $4.200846 \times 10^{13}$ & $4.293402 \times 10^{13}$ & $4.293402 \times 10^{13}$ & $4.293402 \times 10^{13}$ \\
\hline \hline
\end{tabular}

TABLE I. Moments $M_{n}$ for the distribution $j=15 / 2, N=7$ as a function of the number of terms kept in the series (V.7). Odd-order values are obtained using a finite sum.

An interesting consequence of this formula is an approximation for the useful quantity $M_{0}=\sum_{J \geq 0} Q(J)$, representing the total number of levels. Recently, a calculation of $M_{0}$ using fractional parentage coefficients was proposed for $j^{3}$ and $j^{4}$ configurations [22]. In the former case, it was found that $M_{0}=\left(4 j^{2}-1\right) / 8$, and in the latter an expression of $M_{0}$ as a polynomial in $j$ plus a triple sum of products of $6 j$ coefficients was obtained. To our knowledge, no simple analytical expression of the total number of levels is available for a general configuration, even with a single subshell. One has

$$
\begin{aligned}
M_{0} & =\sum_{J \geq 0} Q(J) \simeq \frac{G}{(2 \pi)^{1 / 2} \sigma} \sum_{m \geq 0} \frac{2^{m} \Gamma(1 / 2)}{\Gamma(1 / 2-m)} d_{2 m+1} \\
& =\frac{G}{(2 \pi)^{1 / 2} \sigma}\left(1+\sum_{m>0}(-1)^{m}(2 m-1) ! ! d_{2 m+1}\right) \\
& =\frac{G}{(2 \pi)^{1 / 2} \sigma}\left[1-\frac{1}{24 \sigma^{2}}+\left(\frac{1}{640 \sigma^{4}}+\frac{\kappa_{4}}{8 \sigma^{4}}\right)-\left(\frac{1}{21504 \sigma^{6}}+\frac{5 \kappa_{4}}{192 \sigma^{6}}+\frac{\kappa_{6}}{48 \sigma^{6}}\right)+\cdots\right]
\end{aligned}
$$


where $(2 m-1) ! !=1.3 .5 \cdots(2 m-1)$ is the double factorial. As mentioned concerning the series (III.7), we do not claim that such expansion is convergent, but it is likely of asymptotic nature. An alternative expression for $M_{0}$ is provided by Eq. (II.1b). One has for even $N$, using paper I results for the Gram-Charlier expansion of $P(M)$,

$$
M_{0}=P(0)=\frac{G}{(2 \pi)^{1 / 2} \sigma}\left(1+\sum_{n>1}(-1)^{n}(2 n-1) ! c_{2 n}\right) \quad(\text { even } N)
$$

where the value $H_{2 n}(0)=(-1)^{n}(2 n) ! / 2^{n} n !=(-1)^{n}(2 n-1) ! !$ has been used. The sum of $Q(J)$ for odd $N$ is more complicated and is written as

$$
M_{0}=P(1 / 2)=\frac{G}{(2 \pi)^{1 / 2} \sigma} \exp \left(-1 / 8 \sigma^{2}\right)\left(1+\sum_{n>1} c_{2 n} H e_{2 n}(1 / 2 \sigma)\right) \quad(\text { odd } N)
$$

The approximations (V.9/V.10) are compared in Tables 1 and $\amalg$ as a function of the number of terms in the expansion. The cutoff index $k_{\max }$ is directly related to the number of terms involved in the sums. It corresponds to the maximum order of the cumulant $\kappa\left(k_{\max }\right)$, for instance if $k_{\max }=4$, cumulants up to $\kappa_{4}$ are accounted for. If the form (V.9) is used, the sum contains $k_{\max } / 2+1$ terms. If the form (V.10) is used, the sum contains $k_{\max } / 2$ terms.

These approximations are first tested in Table凹for the single-subshell configuration $7 / 2^{3}$, for which the exact value is $M_{0}=6$. We note that the error slowly decreases with $k_{\max }$, and that both forms oscillate. A rather good approximation is reached with 4 terms included in the sum. As a second example we consider the two-subshell configuration $5 / 2^{2} 37 / 2$, for which the populations $Q(J)$ are plotted in Fig. 2(a). As seen on Table II both approximations are of the same quality whatever $k_{\max }$. Taking only 3 terms - up to the kurtosis $\kappa_{4}$ - provides a poor approximation of the sum $\sum_{J} Q(J)$. With one more term the approximation improves, but this behavior is particular to this case. As a rule, for configurations with a small number of electrons, one notes that the error for both forms oscillates with $k_{\max }$ and tends to decrease, though quite slowly. It appears from this table that about 30 terms are needed to get an accuracy well below the percent level. In Table III, case 3 is the configuration composed of half-filled subshells $j_{i}=i-1 / 2, N_{i}=i$ with $i$ from 1 to 5 . It appears that the second form, involving the Gram-Charlier expression for $P\left(J_{\min }\right)$, provides a much better approximation for this sum. This is probably due to the inaccuracy added by replacing the difference $P(J)-P(J+1)$ by a derivative in defining (V.9). One notices that the accuracy Eq. (V.9) reaches a plateau at $\simeq 0.002$ while the error using Eq. (V.10) decreases. One notes that the approximation labeled as $k_{\max }=4$, which is the first term involving the excess kurtosis $\kappa_{4}$, can be significantly improved by adding more terms in the series if the second form (V.10) is used. The same table presents the results for the 10-subshell configuration corresponding to Fig. 4 analysis. The conclusions obtained in Case-3 configuration apply here too.

\begin{tabular}{cccccccccc}
\hline \hline & \multicolumn{4}{c}{ Case 1 } & & \multicolumn{4}{c}{ Case 2 } \\
\cline { 2 - 4 } \cline { 8 - 10 }$k_{\max }$ & Form 1 & error & Form 2 & error & & Form 1 & error & Form 2 & error \\
\hline 0 & 6.66073 & $1.10(-1)$ & 6.58713 & $9.79(-2)$ & & 20.3458 & $3.56(-1)$ & 20.3254 & $3.55(-1)$ \\
2 & 6.63606 & $1.06(-1)$ & 6.58713 & $9.79(-2)$ & & 20.3390 & $3.56(-1)$ & 20.3254 & $3.55(-1)$ \\
4 & 6.21430 & $3.57(-2)$ & 6.18842 & $3.14(-2)$ & & 17.5044 & $1.67(-1)$ & 17.5050 & $1.67(-1)$ \\
6 & 6.05749 & $9.58(-3)$ & 6.03639 & $6.07(-3)$ & & 14.9108 & $-5.94(-3)$ & 14.9249 & $-5.01(-3)$ \\
8 & 6.10829 & $1.80(-2)$ & 6.07842 & $1.31(-2)$ & & 13.2786 & $-1.15(-1)$ & 13.3013 & $-1.13(-1)$ \\
10 & 6.22233 & $3.71(-2)$ & 6.18026 & $3.00(-2)$ & & 12.6002 & $-1.60(-1)$ & 12.6255 & $-1.58(-1)$ \\
12 & 6.30396 & $5.07(-2)$ & 6.25445 & $4.24(-2)$ & & 12.6392 & $-1.57(-1)$ & 12.6615 & $-1.56(-1)$ \\
16 & 6.27282 & $4.55(-2)$ & 6.23351 & $3.89(-2)$ & & 13.8113 & $-7.92(-2)$ & 13.8172 & $-7.89(-2)$ \\
24 & 6.01031 & $1.72(-3)$ & 6.01452 & $2.42(-3)$ & & 15.9746 & $6.50(-2)$ & 15.9508 & $6.34(-2)$ \\
32 & 6.13742 & $2.29(-2)$ & 6.10293 & $1.72(-2)$ & & 15.8600 & $5.73(-2)$ & 15.8438 & $5.63(-2)$ \\
64 & 5.86148 & $-2.31(-2)$ & 5.94881 & $-8.53(-3)$ & & 14.9298 & $-4.68(-3)$ & 14.9297 & $-4.69(-3)$ \\
128 & 5.98820 & $-1.97(-3)$ & 5.97753 & $-3.74(-3)$ & 15.0072 & $4.77(-4)$ & 15.0065 & $4.33(-4)$ \\
\hline \hline
\end{tabular}

TABLE II. Values of the moment $M_{0}=\sum_{J>0} Q(J)$ using formulas V.9|V.10 as Form 1, Form 2 respectively. The error is the relative variation $M_{0}$ (approx) $/ M_{0}$ (exact) -1 . The value $k_{\max }$ for each row corresponds to the cutoff index (see text for details). Case 1 is the single-subshell configuration $7 / 2^{3}$, for which $M_{0}=6$. Case 2 is the configuration with 2 subshells $5 / 2^{2} 37 / 2^{1}$, for which the exact value is $M_{0}=15$. The notation $a(-b)$ stands for $a \times 10^{-b}$. 


\begin{tabular}{|c|c|c|c|c|c|c|c|c|}
\hline \multirow[b]{2}{*}{$k_{\max }$} & \multicolumn{4}{|c|}{ Case 3} & \multicolumn{4}{|c|}{ Case 4} \\
\hline & Form 1 & error & Form 2 & error & Form 1 & error & Form 2 & error \\
\hline 0 & 260354.3 & $2.36(-2)$ & 259582.2 & $2.06(-2)$ & 131285863 & $2.80(-2)$ & 131285863 & $2.80(-2)$ \\
\hline 2 & 260096.6 & $2.26(-2)$ & 259582.2 & $2.06(-2)$ & 131242959 & $2.77(-2)$ & 131285863 & $2.80(-2)$ \\
\hline 4 & 254912.7 & $2.19(-3)$ & 254474.8 & $4.71(-4)$ & 127833224 & $9.86(-4)$ & 127876115 & $1.32(-3)$ \\
\hline 6 & 254232.3 & $-4.82(-4)$ & 253783.3 & $-2.25(-3)$ & 127175754 & $-4.16(-3)$ & 127213074 & $-3.87(-3)$ \\
\hline 8 & 254664.9 & $1.22(-3)$ & 254199.8 & $-6.10(-4)$ & 127462387 & $-1.92(-3)$ & 127498193 & $-1.64(-3)$ \\
\hline 10 & 254892.6 & $2.11(-3)$ & 254423.7 & $2.70(-4)$ & 127705777 & $-1.19(-5)$ & 127742424 & $2.75(-4)$ \\
\hline 12 & 254885.8 & $2.09(-3)$ & 254419.6 & $2.54(-4)$ & 127754702 & $3.71(-4)$ & 127792226 & $6.65(-4)$ \\
\hline 16 & 254801.5 & $1.76(-3)$ & 254338.3 & $-6.58(-5)$ & 127665972 & $-3.24(-4)$ & 127703495 & $-2.98(-5)$ \\
\hline 24 & 254823.8 & $1.84(-3)$ & 254359.2 & $1.66(-5)$ & 127673721 & $-2.63(-4)$ & 127711002 & $2.90(-5)$ \\
\hline 32 & 254818.4 & $1.82(-3)$ & 254354.1 & $-3.71(-6)$ & 127669135 & $-2.99(-4)$ & 127706465 & $-6.56(-6)$ \\
\hline 64 & 254819.1 & $1.82(-3)$ & 254354.8 & $-9.53(-7)$ & 127670125 & $-2.91(-4)$ & 127707441 & $1.08(-6)$ \\
\hline
\end{tabular}

TABLE III. Values of the moment $M_{0}=\sum_{J \geq 0} Q(J)$ using formulas V.9V.10. Case 3 is the configuration with 5 half-filled subshells $1 / 2^{1} 3 / 2^{2} 5 / 2^{3} 7 / 2^{4} 9 / 2^{5}$, the exact value of the moment is $M_{0}=254355$. Case 4 is the configuration with 10 subshells $j_{i}=1 / 2,3 / 2 \ldots 19 / 2$ all singly populated, with exact value $M_{0}=127707302$. See Table $\amalg$ for details.

\section{NUMBER OF LINES IN A TRANSITION ARRAY}

Among useful applications of the counting of levels with a given total angular momentum $J$, one finds for instance the numbering of Auger amplitudes, for which Kyniène et al obtained a fair approximation [23], or the determination of the number of radiative transitions between two relativistic configurations. We deal here with the case of dipolar transitions (E1 or M1) between two relativistic configurations $A$ and $B$. The number of such lines is (see, e.g. Ref. 2])

$$
N_{A B}=\sum_{J}^{\prime}\left(Q_{A}(J) Q_{B}(J)+Q_{A}(J) Q_{B}(J+1)+Q_{A}(J+1) Q_{B}(J)\right)
$$

where $Q_{A}(J)$ (resp. $Q_{B}(J)$ ) is the number of levels with total angular momentum $J$ in configuration $A$ (resp. $B$ ). The prime in the sum reflects the fact that it is restricted to non-negative $J$ values, and that one must eliminate the $0-0$ transitions if the total number of electrons is even, therefore one has to subtract $Q_{A}(0) Q_{B}(0)$ from the sum. A series of

\begin{tabular}{|c|c|c|c|c|c|c|c|c|c|c|}
\hline \multirow[b]{2}{*}{$k_{\max }$} & \multicolumn{2}{|c|}{ Case 1} & \multicolumn{2}{|c|}{ Case 2} & \multicolumn{2}{|c|}{ Case 3} & \multicolumn{2}{|c|}{ Case 4} & \multicolumn{2}{|c|}{ Case 5} \\
\hline & Count & error & Count & error & Count & error & Count & error & Count & error \\
\hline Exact & 106 & & 79 & & 189 & & 10983 & & 3925292 & \\
\hline 1 & 121.2746 & $1.44(-1)$ & 46.01977 & $-4.18(-1)$ & 222.9916 & $1.80(-1)$ & 12118.94 & $1.03(-1)$ & 4260164 & $8.53(-2)$ \\
\hline 3 & 120.6034 & $1.37(-1)$ & 45.97171 & & 19.6235 & $1.62(-1)$ & 12060.55 & $9.81(-2)$ & 4237309 & \\
\hline 5 & 105.8427 & $-1.48(-3)$ & 33.53130 & & & & & $7.46(-3)$ & 3947621 & \\
\hline 7 & 103.8143 & $-2.06(-2)$ & 41.57908 & & 186.4600 & & 10945.10 & $-3.45(-3)$ & 3911716 & \\
\hline 9 & & $-1.97(-2)$ & 58.53235 & & & & 10969.21 & $-1.26(-3)$ & 3920 & \\
\hline 15 & 104.4607 & $-1.45(-2)$ & 62.29300 & $-2.12(-1)$ & 187.5295 & & 10986.25 & $2.96(-4)$ & 3925469 & $4.51(-5)$ \\
\hline 23 & 104.6794 & $-1.24(-2)$ & 69.30309 & $-1.23(-1)$ & & $-7.79(-3)$ & 10985.39 & $2.18(-4)$ & 3925279 & $-3.41(-6)$ \\
\hline 31 & 104.4558 & $-1.45(-2)$ & 75.71697 & $-4.16(-2)$ & 187.5797 & $-7.52(-3)$ & 10985.35 & $2.14(-4)$ & 3925281 & $-2.72(-6)$ \\
\hline 47 & 104.3896 & $-1.51(-2)$ & 77.91521 & $-1.37(-2)$ & 187.7243 & $-6.75(-3)$ & 10985.36 & $2.15(-4)$ & 3925283 & $-2.37(-6)$ \\
\hline 63 & 104.3722 & $-1.53(-2)$ & 76.75403 & $-2.84(-2)$ & 188.2175 & $-4.14(-3)$ & 10985.39 & $2.18(-4)$ & 3925284 & $-1.97(-6)$ \\
\hline 127 & 104.7995 & $-1.13(-2)$ & 77.78638 & $-1.54(-2)$ & 191.8610 & $1.51(-2)$ & 10985.43 & $2.21(-4)$ & 3925288 & $-9.46(-7)$ \\
\hline
\end{tabular}

TABLE IV. Number of lines obtained with the Gram-Charlier-like expansion as a function of the cutoff index $k_{\max }$ for various transition arrays. Case 1: $9 / 2^{3} \rightarrow 9 / 2^{2} 11 / 2$, case $2: 5 / 2^{2} 37 / 2 \rightarrow 5 / 2^{2} 39 / 2$, case $3: 3 / 2^{2} 5 / 2^{2} \rightarrow 3 / 2^{2} 5 / 2^{1} 7 / 2^{1}$, case 4 : $5 / 2^{3} 9 / 2^{3} \rightarrow 5 / 2^{2} 9 / 2^{4}$, case $5: 1 / 2^{1} 3 / 2^{2} 5 / 2^{3} 7 / 2^{4} \rightarrow 1 / 2^{1} 3 / 2^{2} 5 / 2^{3} 7 / 2^{3} 9 / 2^{1}$. The value $k$ is the maximum index $2 m+1$ accounted for in the expansion (III.7) and the error is the relative variation on the line number. The notation $a(-b)$ stands for $a \times 10^{-b}$.

examples is provided in the table IV for various pairs of relativistic configurations. The number of lines obtained with 
the explicit sum (VI.1) is compared to the exact value as a function of the cutoff index $k_{\max }$. To put it differently, the number of terms retained in Gram-Charlier-like expansion for both configurations is $\left(k_{\max }+1\right) / 2$. The conclusions drawn for the angular momentum distribution may be reiterated here. In the simple case $9 / 2^{3} \rightarrow 9 / 2^{2} 11 / 2$, the three-term expansion involving cumulants up to the kurtosis and used in Ref. [2] provides a fair approximation, which is not significantly improved by adding many more terms. In the special case $5 / 2^{2} 37 / 2 \rightarrow 5 / 2^{2} 39 / 2$, for which we have seen that the kurtosis-based approximation fails, the same conclusions hold here and a large number of terms are required in order to get an acceptable number of lines. In the case $3 / 2^{2} 5 / 2^{2} \rightarrow 3 / 2^{2} 5 / 2^{1} 7 / 2^{1}$, little improvement is brought by adding terms beyond the third $\left(k_{\max }=5\right)$. Similar conclusions hold in the case $5 / 2^{3} 9 / 2^{3} \rightarrow 5 / 2^{2} 9 / 2^{4}$. One notes that a better accuracy is achieved when more electrons are involved. Finally in the multiple-subshell case $1 / 2^{1} 3 / 2^{2} 5 / 2^{3} 7 / 2^{4} \rightarrow 1 / 2^{1} 3 / 2^{2} 5 / 2^{3} 7 / 2^{3} 9 / 2^{1}$, one notes a regular increase of the accuracy provided by the expansion, the first three terms providing an accuracy below the percent level. Finally it is worth mentioning that increasing $k_{\max }$ to very high values - which depend on the pair of configurations analyzed - one notes that the expansion begins to diverge, in agreement with the observation done on the number of levels $Q(J)$. As mentioned previously, the expansion derived here is asymptotic, and the larger the total number of electrons the better the approximation.

\section{CONCLUSION}

The generating function previously obtained for the distribution of the magnetic quantum number $M$ has been used to derive properties on the distribution of the total angular momentum $J$. It has been shown that in any single-subshell relativistic configuration this distribution can be efficiently computed using recurrence relations, and that even-odd staggering can be described for a single subshell with an even number of electrons. The analysis on the magnetic-quantum-number distribution allows us to obtain an expression for the $J$-distribution that generalizes the Bethe formula in the form of a Gram-Charlier-like expansion. This expansion has been tested in a series of cases and proved to be efficient, even in most cases when few terms are accounted for. However, as for the magneticquantum-number-distribution analysis, we observe the Gram-Charlier-like expansion is probably not convergent but of asymptotic nature. The formulas obtained also provide accurate approximations for the total number of levels in a relativistic configuration and for the number of lines in a transition array.

\section{Appendix A: Description of the even-odd staggering in the angular-momentum distribution using the generating function}

As pointed out earlier [19], the generating function technique is also useful when analyzing the predominance of the levels with even $J$ values. From definition (II.6) and the symmetry property on $Q(J)$ (II.2), one has for a single-subshell configuration with an even number of electrons $N$

$$
\begin{aligned}
\mathscr{G}(j, N ;-1)= & (-1)^{J_{\max }+1}\left[Q(0)-Q(1)+Q(2) \cdots+(-1)^{J_{\max }} Q\left(J_{\max }\right)\right] \\
& +(-1)^{J_{\max }}\left[Q(-1)-Q(-2)+Q(-3) \cdots+(-1)^{J_{\max }} Q\left(-J_{\max }-1\right)\right] \\
= & -2 \mathscr{E}
\end{aligned}
$$

where $\mathscr{E}$ is the even-odd excess

$$
\mathscr{E}=\sum_{n \geq 0} Q(2 n)-\sum_{n \geq 0} Q(2 n+1) .
$$

We have used the value $J_{\max }=N(2 j+1-N) / 2$ from which $(-1)^{J_{\max }+1}=-1$ if $N$ is even. Therefore the evaluation of the excess is directly related to $\mathscr{G}(j, N ;-1)$. Furthermore for a multi-subshell configuration, the above relation still holds while the product in (II.6) is replaced by the product of the contribution of every subshell. The evaluation of the excess is therefore reduced to computing the product (II.6) at $z=-1$. To this respect, one notices that this quantity involves both at the numerator and the denominator $N / 2$ factors of the form $\left(z^{2 r}-1\right)$ which vanish at $z=-1$. The indeterminacy is removed by the de L'Hospital rule which amounts to replace these factors by their derivative. Namely one has, after eliminating factors with odd powers,

$$
\begin{aligned}
\mathscr{E} & =-\frac{1}{2} \mathscr{G}(j, N ;-1)=\lim _{z \rightarrow-1} \prod_{r=1}^{N / 2} \frac{z^{2 j+3-2 r}-1}{z^{2 r}-1} \\
& =\prod_{r=1}^{N / 2} \frac{2 j+3-2 r}{2 r}=\left(\begin{array}{c}
j+1 / 2 \\
N / 2
\end{array}\right)
\end{aligned}
$$


in agreement with Ref. [18. In the case where $N$ is odd, no simple relation like A.1 holds. However, a direct computation of the $Q(J)$ distribution allows one to check that the staggering on $Q(J)$ between $J=2 n+1 / 2$ and $J=2 n+3 / 2$ is much less important: one may, e.g., look at results presented in subsection IV C. The same formalism also applies for any multi-subshell relativistic configuration with an even number of electrons. Then, Eq. A.1 holds provided $\mathscr{G}(j, N ; z)$ is replaced by $(z-1)$ multiplied by the product of the $\mathscr{F}\left(j_{s}, N_{s} ; z\right)$, $\mathscr{F}$ being defined by Eq. (II.4). If every subshell has an even number of electrons, due to this factorization property, the excess is simply given by

$$
\mathscr{E}=\prod_{s=1}^{w}\left(\begin{array}{c}
j_{s}+1 / 2 \\
N_{s} / 2
\end{array}\right) \text { if all } N_{s} \text { even }
$$

while $\mathscr{E}=0$ if at least one of the subshells has an odd occupation number. This last point is easy to verify. Let us consider a factor $\mathscr{F}\left(j_{s}, N_{s} ; z\right)$ with $N_{s}$ odd. From the definition (II.4), the denominator contains $\left(N_{s}-1\right) / 2$ factors with an even power $z^{2 r}-1$, while there are $\left(N_{s}+1\right) / 2$ such factors in the numerator. When evaluating such product at $z=-1$, the result will be zero.

One may also generalize the above derivation to the formal case where $j$ is integer. In this case the total momentum $J$ is integer, and the excess can be computed whatever $N$. Noting that $J_{\max }=N(2 j+1-N) / 2$ has the parity of $N / 2$ for even $N$ and of $(2 j+1-N) / 2$ for odd $N$, the same method as above provides the excess value

$$
\mathscr{E}= \begin{cases}(-1)^{N / 2}\left(\begin{array}{c}
j \\
N / 2
\end{array}\right) & \text { for even } N \\
(-1)^{(2 j+1-N) / 2}\left(\begin{array}{c}
j \\
(N-1) / 2
\end{array}\right) & \text { for odd } N\end{cases}
$$

which indicates that the excess never vanishes. For a multi-subshell configuration, the same argument as above proves that the excess is given by the product of the individual contributions (A.5). The excess never vanishes in this case too.

\section{Appendix B: Alternative expression for the Gram-Charlier-like expansion}

The expression (III.11a) for the coefficients of the Gram-Charlier-like expansion is formally simple. However from a computational point of view it requires the definition of the partitions of the even integer $2 m$ which becomes rather time-consuming for large $m$ since the number of such partitions increases exponentially with $m^{1 / 2}$ as shown by the Ramanujan-Hardy formula. An alternative formulation uses the moments of the $P(M)$ distribution

$$
\mu_{k}=\sum_{M} M^{k} P(M) / \sum_{M} P(M) .
$$

Such moments can be straightforwardly derived from the expression of the cumulants using the well-known formula [24]

$$
\mu_{2 k}=\kappa_{2 k}+\sum_{m=1}^{k-1}\left(\begin{array}{c}
2 k-1 \\
2 m-1
\end{array}\right) \kappa_{2 m} \mu_{2 k-2 m}
$$

which accounts here for the fact that the $P(M)$ distribution is symmetric, so that odd-order moments vanish. The cumulants for the $P(M)$ distribution are given by Eq. (1I.10). From the relation (III.8) and from the standard equation providing the Gram-Charlier coefficients as a function of the moments,

$$
c_{2 n}=\sum_{j=0}^{n} \frac{(-1)^{j} \mu_{2 n-2 j} / \sigma^{2 n-2 j}}{2^{j} j !(2 n-2 j) !}
$$


one gets, with the variable change $q=p+j$ so that $n-j=m-q$, and using the Chebyshev-Hermite polynomial expansion (III.3),

$$
\begin{aligned}
d_{2 m+1} & =\sum_{\substack{n, p, j \\
n+p=m}} \frac{(2 \sigma)^{-2 p}}{(2 p+1) !} \frac{(-1)^{j} \mu_{2 n-2 j} / \sigma^{2 n-2 j}}{2^{j} j !(2 n-2 j) !} \\
& =\sum_{\substack{n, q, j \\
n+q-j=m}} \frac{(2 \sigma)^{2 j-2 q}}{(2 q-2 j+1) !} \frac{(-1)^{j} \mu_{2 m-2 q} / \sigma^{2 m-2 q}}{2^{j} j !(2 m-2 q) !} \\
& =\sum_{q=0}^{m} \frac{2 \sigma}{(2 m-2 q) !} \frac{\mu_{2 m-2 q}}{\sigma^{2 m-2 q}} \sum_{j=0}^{q} \frac{(-1)^{j}(2 \sigma)^{2 j-2 q-1}}{2^{j} j !(2 q+1-2 j) !} \\
& =2 \sigma \sum_{q=0}^{m} \frac{\mu_{2 m-2 q} / \sigma^{2 m-2 q}}{(2 m-2 q) !(2 q+1) !} H e_{2 q+1}\left((2 \sigma)^{-1}\right) \\
& =\frac{2 \sigma}{(2 m+1) !} \sum_{q=0}^{m}\left(\begin{array}{c}
2 m+1 \\
2 q+1
\end{array}\right) H e_{2 q+1}\left((2 \sigma)^{-1}\right) \frac{\mu_{2 m-2 q}}{\sigma^{2 m-2 q}} .
\end{aligned}
$$

This expression is almost as simple as (B.3) since the Hermite polynomials can be computed very efficiently using simple recurrence relations. The first coefficients are

$$
\begin{aligned}
& d_{3}=\frac{1}{24 \sigma^{2}} \\
& d_{5}=\frac{\mu_{4}}{24 \sigma^{4}}+\frac{1}{1920 \sigma^{4}}-\frac{1}{8} \\
& d_{7}=\frac{\mu_{6}}{720 \sigma^{6}}+\frac{\mu_{4}}{576 \sigma^{6}}-\frac{\mu_{4}}{48 \sigma^{4}}+\frac{1}{322560 \sigma^{6}}-\frac{1}{192 \sigma^{2}}+\frac{1}{24} .
\end{aligned}
$$

\section{Appendix C: Gram-Charlier-like analysis for a subshell with several electrons}

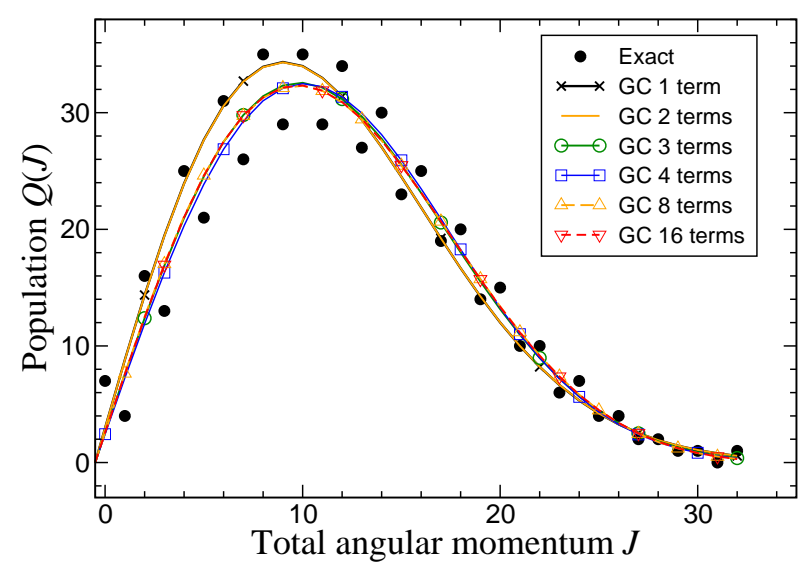

(a) Configuration $j_{1}=15 / 2, N_{1}=8$

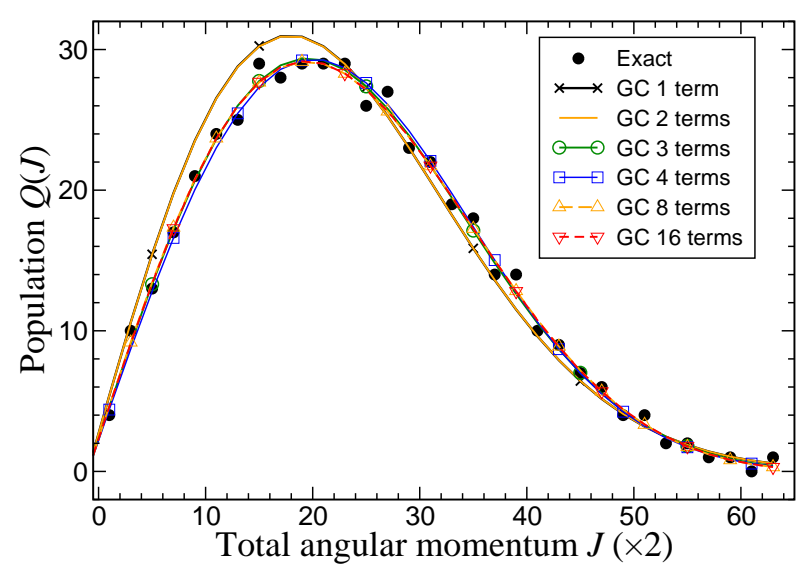

(b) Configuration $j_{1}=15 / 2, N_{1}=7$

FIG. 5. Exact and Gram-Charlier-like approximation for the total angular momentum distribution $Q(J)$ in the relativistic configuration with one subshell $j_{1}=15 / 2, N_{1}=8$ or 7 .

We consider in Fig. 5(a) the $J$ distribution in the case of a single subshell $j=15 / 2, N=8$. One notices a rough agreement with the Gram-Charlier-like expansion, even with few terms. However the odd-even staggering noticed by Bauche and Cossé [18, 19] and revisited in Appendix $₫$ is not reproduced even with a large number of terms. We observe that the expansion proposed here provides a good agreement with the distribution "averaged" on even and odd cases. 
Finally Fig. $5(\mathrm{~b})$ presents the $J$ distribution for the single-subshell configuration $j=15 / 2, N=7$. The GramCharlier-like expansion provides an acceptable approximation when only 3 terms are accounted for, with little improvement brought by a greater number of terms. One notes that some staggering is observed again between the angular momenta $J=2 n+1 / 2$ and $J=2 n-1 / 2$, though this effect is smaller than the even-odd variation observed for an even number of electrons.

[1] S. A. Moszkowski, Some statistical properties of level and line distributions in atomic spectra, Tech. Rep. (RAND Corp., Santa Monica, California, 1960).

[2] J. Bauche and C. Bauche-Arnoult, Level and line statistics in atomic spectra, J. Phys. B: At. Mol. Opt. Phys. 20, 1659 (1987)

[3] F. Gilleron and J.-C. Pain, Efficient methods for calculating the number of states, levels and lines in atomic configurations, High Energy Density Phys. 5, 320 (2009).

[4] Q. Porcherot, J.-C. Pain, F. Gilleron, and T. Blenski, A consistent approach for mixed detailed and statistical calculation of opacities in hot plasmas, High Energy Density Phys. 7, 234 (2011).

[5] J.-C. Pain and F. Gilleron, Accounting for highly excited states in detailed opacity calculations, High Energy Density Phys. 15, 30 (2015).

[6] C. A. Iglesias and V. Sonnad, Partially resolved transition array model for atomic spectra, High Energy Density Phys. 8, 154 (2012).

[7] C. A. Iglesias, Statistical line-by-line model for atomic spectra in intermediate coupling, High Energy Density Phys. 8, 253 (2012).

[8] C. A. Iglesias, Partially resolved transition array model in intermediate coupling, High Energy Density Phys. 8, 260 (2012).

[9] B. G. Wilson, C. A. Iglesias, and M. H. Chen, Partially resolved super transition array method, High Energy Density Phys. 14, 67 (2015).

[10] Y. Kurzweil and G. Hazak, Summation of the spectra of all partially resolved transition arrays in a supertransition array, Phys. Rev. E 94, 053210 (2016).

[11] J.-C. Pain, F. Gilleron, J. Bauche, and C. Bauche-Arnoult, Statistics of electric-quadrupole lines in atomic spectra, J. Phys. B: At. Mol. Opt. Phys. 45, 135006 (2012)

[12] J.-C. Pain and M. Poirier, Analytical and numerical expressions for the number of atomic configurations contained in a supershell, J. Phys. B: At. Mol. Opt. Phys. 53, 115002 (2020).

[13] H. A. Bethe, An attempt to calculate the number of energy levels of a heavy nucleus, Phys. Rev. 50, 332 (1936).

[14] J. P. Elliott and A. M. Lane, The nuclear shell-model, in Structure of Atomic Nuclei / Bau der Atomkerne (Springer, Berlin, Heidelberg, 1957) pp. 241-410.

[15] S. A. Moszkowski, Models of nuclear structure, in Structure of Atomic Nuclei / Bau der Atomkerne (Springer Berlin Heidelberg, Berlin, Heidelberg, 1957) pp. 411-550.

[16] M. Hirst and B. G. Wybourne, Statistical group theory and the distribution of angular momentum states: II, J. Phys. A: Math. Gen. 19, 1545 (1986).

[17] M. Poirier and J.-C. Pain, Angular momentum distribution in a relativistic configuration: Magnetic quantum number analysis, submitted (2021).

[18] J. Bauche and P. Cossé, Odd-even staggering in the $\mathrm{J}$ and $\mathrm{L}$ distributions of atomic configurations, J. Phys. B: At. Mol. Opt. Phys. 30, 1411 (1997)

[19] J.-C. Pain, Regularities and symmetries in atomic structure and spectra, High Energy Density Phys. 9, 392 (2013)

[20] M. Abramowitz and I. Stegun, Handbook of Mathematical Functions (National Bureau of Standards, Washington DC, USA, 1972).

[21] A. Erdélyi, Asymptotic Expansions, Dover Books on Mathematics (Dover Publications, 1956).

[22] J.-C. Pain, Total number of $j$ levels for identical particles in a single- $j$ shell using coefficients of fractional parentage, Phys. Rev. C 99, 054321 (2019).

[23] A. Kyniène, R. Karazija, and V. Jonauskas, Statistical properties of Auger amplitudes and rates, J. Electron Spectrosc. Relat. Phenom. 122, 181 (2002)

[24] A. Stuart and J. K. Ord, Kendall's Advanced Theory of Statistics - Distribution Theory, Vol. 1 (John Wiley and Sons, London UK, 1994). 